\title{
Traveling Wave Solutions of the Kadomtsev-Petviashvili-Benjamin-Bona-Mahony Equation
}

\author{
Zhengyong Ouyang \\ Department of Mathematics, Foshan University, Foshan, Guangdong 528000, China \\ Correspondence should be addressed to Zhengyong Ouyang; zyouyang_math@163.com
}

Received 26 March 2014; Accepted 17 April 2014; Published 18 June 2014

Academic Editor: Junling Ma

Copyright (C) 2014 Zhengyong Ouyang. This is an open access article distributed under the Creative Commons Attribution License, which permits unrestricted use, distribution, and reproduction in any medium, provided the original work is properly cited.

\begin{abstract}
We use bifurcation method of dynamical systems to study exact traveling wave solutions of a nonlinear evolution equation. We obtain exact explicit expressions of bell-shaped solitary wave solutions involving more free parameters, and some existing results are corrected and improved. Also, we get some new exact periodic wave solutions in parameter forms of the Jacobian elliptic function. Further, we find that the bell-shaped waves are limits of the periodic waves in some sense. The results imply that we can deduce bell-shaped waves from periodic waves for some nonlinear evolution equations.
\end{abstract}

\section{Introduction}

The Benjamin-Bona-Mahony (BBM) equation [1]

$$
u_{t}+u_{x}+u u_{x}-u_{x x t}=0
$$

was proposed as the model for propagation of long waves where nonlinear dispersion is incorporated. The KadomtsevPetviashvili (KP) equation [2]

$$
\left(u_{t}+a u u_{x}+u_{x x x}\right)_{x}+u_{y y}=0
$$

was given as the generalization of the $\mathrm{KdV}$ equation. In addition, both BBM and $\mathrm{KdV}$ equations can be used to describe long wavelength in liquids, fluids, and so forth. Combining the two equations, the Kadomtsev-PetviashviliBenjamin-Bona-Mahony (KP-BBM) equation

$$
\left(u_{t}+u_{x}-a\left(u^{2}\right)_{x}-b u_{x x t}\right)_{x}+k u_{y y}=0
$$

was presented in [3] for further study. Some methods are developed and applied to find exact solutions of nonlinear evolution equations because exact solutions play an important role in the comprehension of nonlinear phenomena. For instance, extended tanh method, extended mapping method with symbol computation, and bifurcation method of dynamical systems are employed to study (3) [4-6], and some solitary wave solutions and triangle periodic wave solutions were obtained.

However, there is no method which can be applied to all nonlinear evolution equations. The research on the solutions of the KP-BBM equation now appears insufficient. Further studies are necessary for the traveling wave solutions of the KP-BBM equation. The purpose of this paper is to apply the bifurcation method [7-10] of dynamical systems to continue to seek traveling waves of (3). Firstly, we obtain bell-shaped solitary wave solutions involving more free parameters, and some results in [6] are corrected and improved. Then, we get some new periodic wave solutions in parameter forms of Jacobian elliptic function, and numerical simulation verifies the validity of these periodic solutions. The periodic wave solutions obtained in this paper are different from those in [5]. Furthermore, we find an interesting relationship between the bell-shaped waves and periodic waves; that is, the bellshaped waves are limits of the periodic waves in forms of Jacobian elliptic function as modulus approaches 1 .

This paper is organized as follows. First, we draw the bifurcation phase portraits of planar system according to the KP-BBM equation in Section 2. Second, bell-shaped solitary wave solutions to the equation under consideration are presented in Section 3. Third, periodic solitary waves are 
given in the forms of Jacobian elliptic function and numerical simulation is done. Finally, the relationship between the bell-shaped solitary waves and periodic waves is proved in Section 4 .

\section{Bifurcation Phase Portraits of System (6)}

Suppose (3) possesses traveling wave solutions in the form $u(x, t)=\varphi(\xi), \xi=x+r y-c t$, where $c$ is the wave speed and $r$ is a real constant. Substituting $u(x, t)=\varphi(\xi), \xi=x+r y-c t$ into (3) admits to the following ODE:

$$
\left(1+k r^{2}-c\right) \varphi^{\prime \prime}-a\left(\varphi^{2}\right)^{\prime \prime}+b c \varphi^{(4)}=0,
$$

where the derivative is for variable $\xi$. Integrating (4) twice with respect to $\xi$ and letting the first integral constant take value zero, it follows that

$$
\left(1+k r^{2}-c\right) \varphi-a \varphi^{2}+b c \varphi^{\prime \prime}=g
$$

where $g$ is the second integral constant.

Equation (5) is equivalent to the following two-dimensional system:

$$
\frac{\mathrm{d} \varphi}{\mathrm{d} \xi}=y, \quad \frac{\mathrm{d} y}{\mathrm{~d} \xi}=\frac{a \varphi^{2}-\left(1+k r^{2}-c\right) \varphi+g}{b c} .
$$

It is obvious that system (6) has the first integral

$$
H(\varphi, y)=\frac{1}{2} y^{2}-\frac{a}{3 b c} \varphi^{3}+\frac{1+k r^{2}-c}{2 b c} \varphi^{2}-\frac{g}{b c} \varphi=h,
$$

where $h$ is the constant of integration.

Define $\Delta=\left(1+k r^{2}-c\right)^{2}-4 a g$. When $\Delta>0$, there are two equilibrium points $\left(\varphi_{1}, 0\right)$ and $\left(\varphi_{2}, 0\right)$ of $(6)$ on $\varphi$-axis, where $\varphi_{1}=\left(\left(1+k r^{2}-c\right)-\sqrt{\Delta}\right) / 2 a, \varphi_{2}=\left(\left(1+k r^{2}-c\right)+\sqrt{\Delta}\right) / 2 a$. The Hamiltonian $H$ of $\left(\varphi_{1}, 0\right)$ and $\left(\varphi_{2}, 0\right)$ is denoted by $h_{1}=$ $H\left(\varphi_{1}, 0\right)$ and $h_{2}=H\left(\varphi_{2}, 0\right)$.

In the case of $1+k r^{2}-c<0$ and $1+k r^{2}-c>0$, the bifurcation phase portraits of system (6) see Figures 1 and 2 in [6], respectively, in which there are some homoclinic and periodic orbits of system (6). For our purpose, we redraw the homolinic and periodic orbits in this paper(see Figures 1-3).

\section{Exact Explicit Expressions of Solitary Wave Solutions}

In this section, we discuss bell-shaped wave solutions under $g=0$ and $g \neq 0$, respectively.

\subsection{The Case $g=0$. System (6) can be rewritten as}

$$
\frac{\mathrm{d} \varphi}{\mathrm{d} \xi}=y, \quad \frac{\mathrm{d} y}{\mathrm{~d} \xi}=\frac{a \varphi^{2}-\left(1+k r^{2}-c\right) \varphi}{b c} .
$$

The first integral of (8) is

$$
H(\varphi, y)=\frac{1}{2} y^{2}-\frac{a}{3 b c} \varphi^{3}+\frac{1+k r^{2}-c}{2 b c} \varphi^{2}=h .
$$

When $\left(1+k r^{2}-c\right) / b c<0$, there are two homoclinic orbits $\Gamma_{1}$ and $\Gamma_{2}$ (see Figures 1(a) and 1(b)). In $\varphi$-y plane, $\Gamma_{1}$ and $\Gamma_{2}$ can be described by

$$
\begin{aligned}
& y^{2}=\frac{2 a}{3 b c} \varphi^{3}-\frac{1+k r^{2}-c}{b c} \varphi^{2}, \\
& \varphi \in\left(0, \varphi^{*}\right) \text { or } \varphi \in\left(\varphi^{*}, 0\right),
\end{aligned}
$$

where $\varphi^{*}=3\left(1+k r^{2}-c\right) / 2 a$. That is,

$$
y= \pm \sqrt{\frac{2 a}{3 b c} \varphi^{3}-\frac{1+k r^{2}-c}{b c} \varphi^{2}} .
$$

Substituting (11) into $\mathrm{d} \varphi / \mathrm{d} \xi=y$ and integrating along homoclinc orbits $\Gamma_{1}$ and $\Gamma_{2}$, respectively, we get

$$
\int_{\varphi}^{\varphi^{*}} \frac{\mathrm{d} s}{\sqrt{(2 a / 3 b c) s^{3}-\left(\left(1+k r^{2}-c\right) / b c\right) s^{2}}}= \pm|\xi| .
$$

Completing the above integration, it follows that

$$
\begin{aligned}
& u_{1}(x, y, t) \\
& =\frac{3\left(1+k r^{2}-c\right)}{a\left[1+\cosh \left(\sqrt{-\left(\left(1+k r^{2}-c\right) / b c\right)}(x+r y-c t)\right)\right]} .
\end{aligned}
$$

Remark 1. $u_{1}(x, y, t)$ is a bright soliton solution when $b c / a<$ 0 and a dark soliton solution when $b c / a>0$. If the real number $r$ in (13) takes value 1, then solution (13) is the same to solution (1.2) in [6]. Solution (1.1) in [6] is not a real solution of the KP-BBM equation; it is obvious that solution (1.1) tends to infinite as $\xi \rightarrow 0$, and it does not satisfy the KP-BBM equation (3).

When $\left(1+k r^{2}+c\right) / b c>0$, there are two homoclinic orbits $\Gamma_{3}$ and $\Gamma_{4}$ (see Figures 2(a) and 2(b)). Similarly solitary wave solutions according to $\Gamma_{3}$ and $\Gamma_{4}$ are obtained as follows:

$$
\begin{aligned}
& u_{2}(x, y, t) \\
& =\left(\left(1+k r^{2}-c\right)\right. \\
& \quad \times\left[-2+\cosh \left(\sqrt{\left.\left.\left.\frac{1+k r^{2}-c}{b c}(x+r y-c t)\right)\right]\right)}\right.\right. \\
& \quad\left(a \left[1+\cosh \left(\sqrt{\left.\left.\left.\frac{1+k r^{2}-c}{b c}(x+r y-c t)\right)\right]\right)^{-1}} .\right.\right.\right.
\end{aligned}
$$

Remark 2. If the real number $r$ in (14) takes value 1 , then solution (14) is the same to solution (1.4) in [6]. Solution (1.5) in [6] is not a real solution of the KP-BBM equation; it is easy to verify that it does not satisfy the KP-BBM equation (3). 


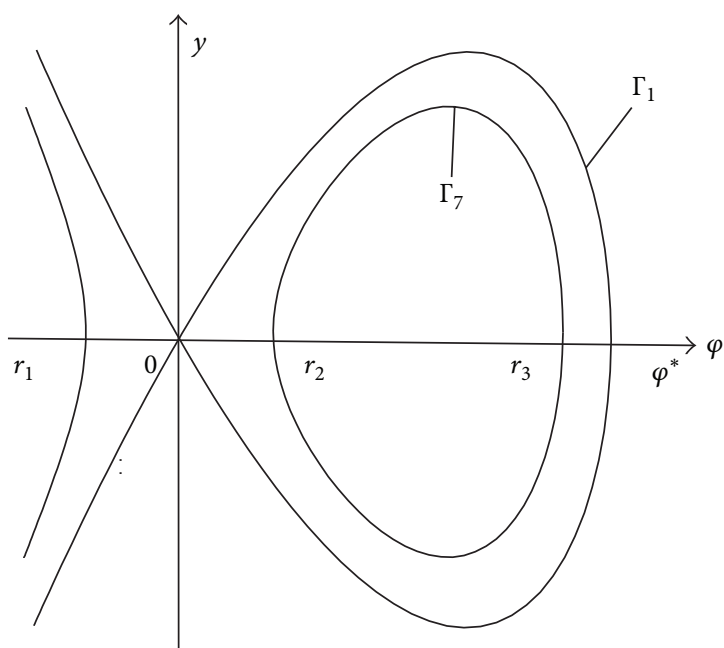

(a) $b c / a<0$

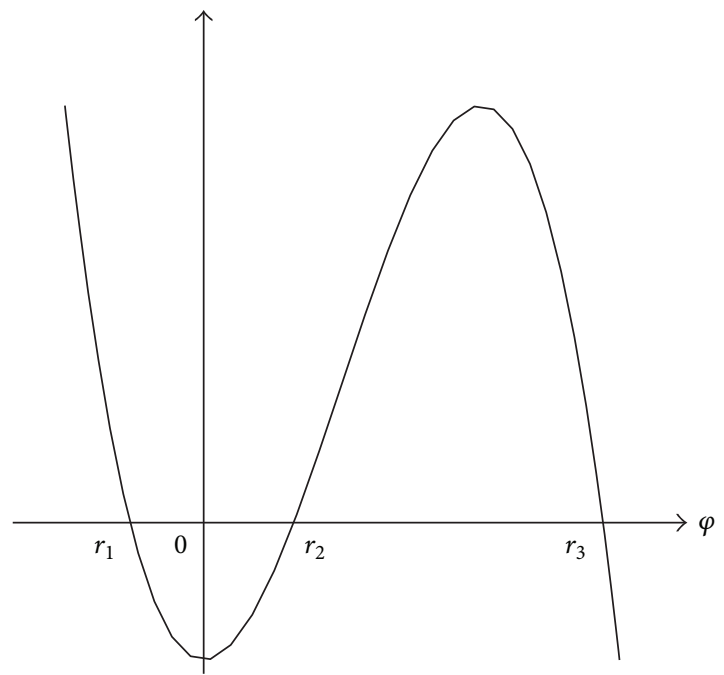

(c)

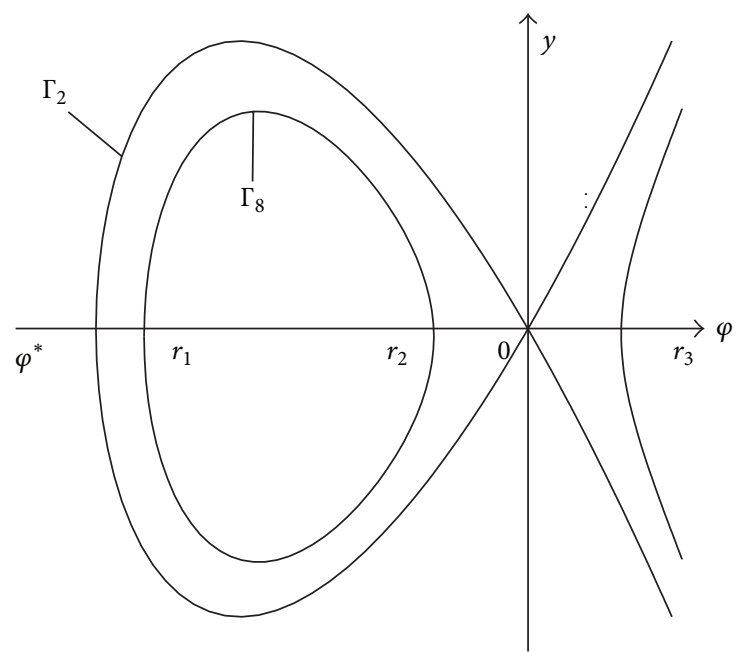

(b) $b c / a>0$

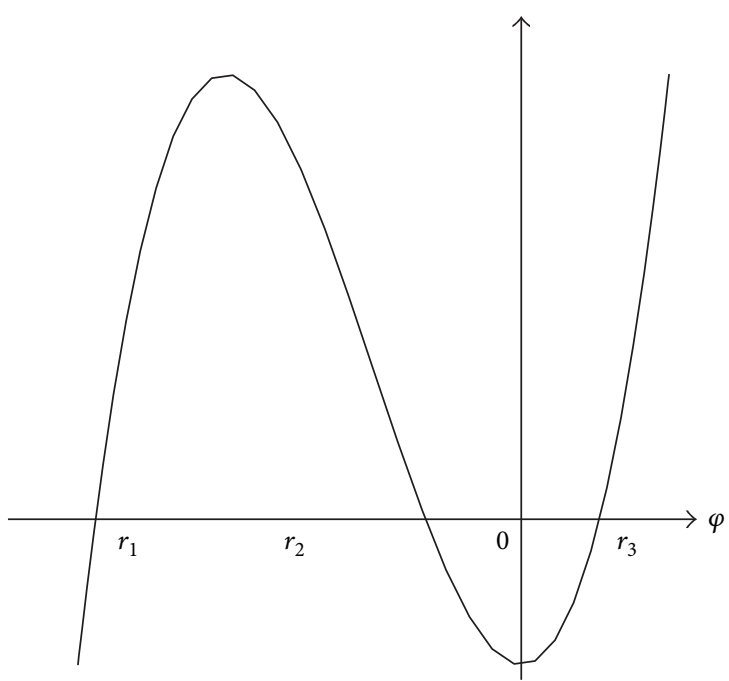

(d)

FIGURE 1: The bifurcation phase portraits of system (6) with $g=0$ and $\left(1+k r^{2}+c\right) / b c<0$.

3.2. The Case $g \neq 0$. There are two homoclinic orbits $\Gamma_{5}$ and $\Gamma_{6}$ when $g \neq 0$ (see Figures 3(a) and 3(b)). $\Gamma_{5}$ and $\Gamma_{6}$ can be described by

$$
y^{2}=\frac{2 a}{3 b c} \varphi^{3}-\frac{1+k r^{2}-c}{b c} \varphi^{2}+\frac{2 g}{b c} \varphi+2 h
$$

When $b c / a<0$, the corresponding homoclinic orbit $\Gamma_{5}$ has a double zero point $\varphi_{1}$ and a zero point $\varphi_{3}$ on $\varphi$-axis (see Figure 3(a)), so (15) can be rewritten as

$$
y^{2}=\frac{2 a}{3 b c}\left(\varphi-\varphi_{1}\right)^{2}\left(\varphi-\varphi_{3}\right)
$$

that is,

$$
y= \pm \sqrt{\frac{2 a}{3 b c}\left(\varphi-\varphi_{1}\right)^{2}\left(\varphi-\varphi_{3}\right)} .
$$

Substituting (17) into $\mathrm{d} \varphi / \mathrm{d} \xi=y$ and integrating along homoclinic orbits $\Gamma_{5}$, we get

$$
\int_{\varphi}^{\varphi_{3}} \frac{\mathrm{d} s}{\sqrt{(2 a / 3 b c)\left(s-\varphi_{1}\right)^{2}\left(s-\varphi_{3}\right)}}=|\xi|,
$$

where $\varphi_{1}=\left(1+k r^{2}-c-\sqrt{\Delta}\right) / 2 a$ and $\varphi_{3}=\left(1+k r^{2}-c+2 \sqrt{\Delta}\right) / 2 a$ if $a>0$ and $b c<0$; then, completing (18) we get the following solution:

$$
\begin{aligned}
& u_{3}(x, y, t) \\
& =\left(\left(1+k r^{2}-c-\sqrt{\Delta}\right)\right. \\
& \quad \times \cosh \sqrt{-\frac{\sqrt{\Delta}}{b c}}(x+r y-c t)+1
\end{aligned}
$$




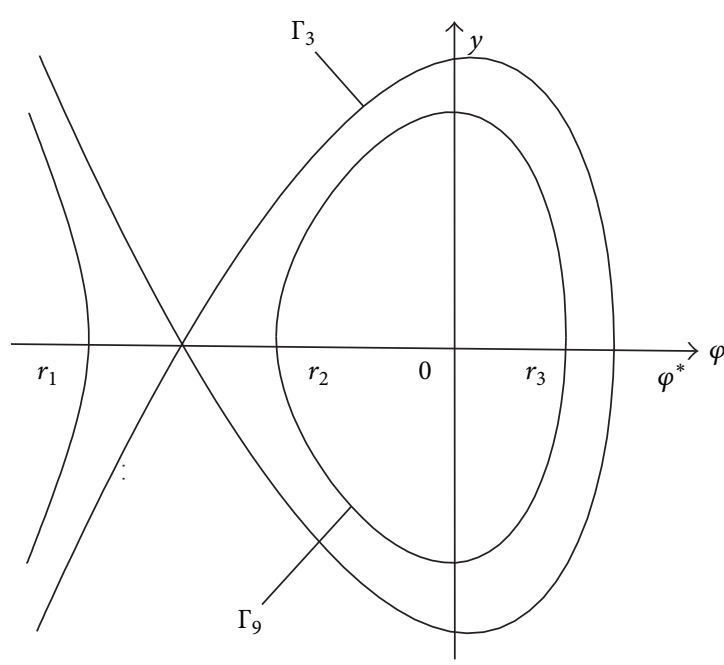

(a) $b c / a<0$

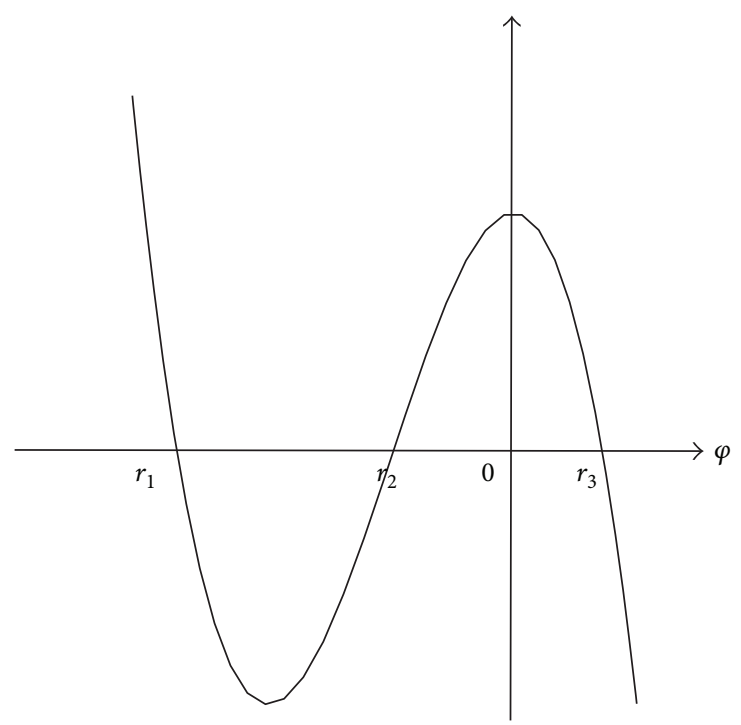

(c)

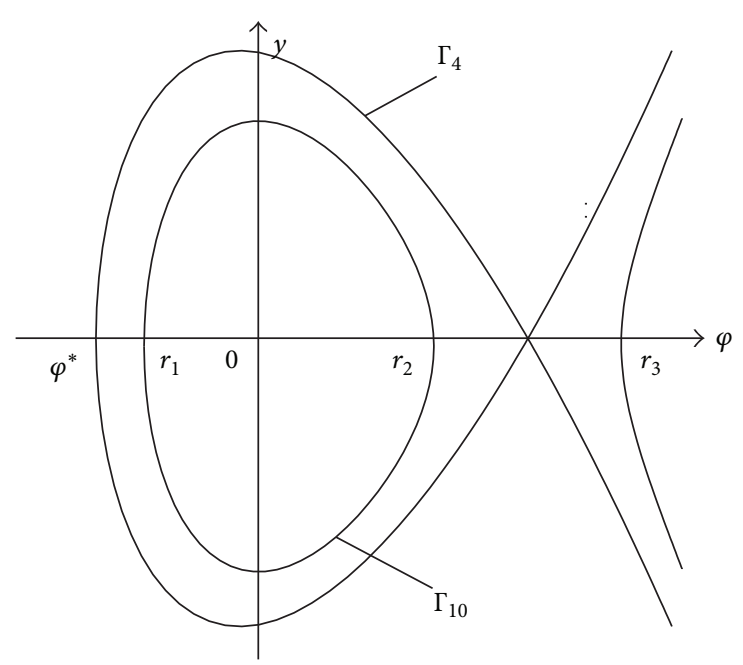

(b) $b c / a>0$

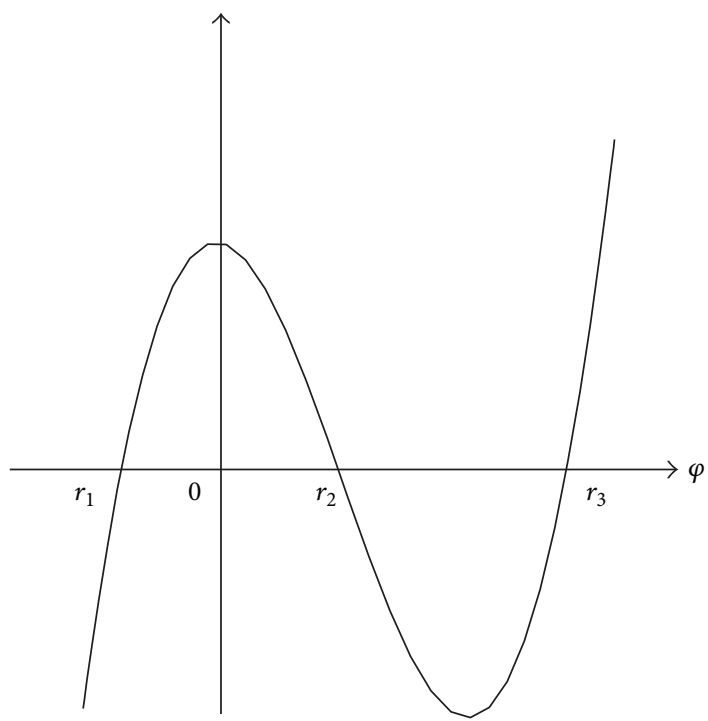

(d)

FIgURE 2: The bifurcation phase portraits of system (6) with $g=0$ and $\left(1+k r^{2}+c\right) / b c>0$.

$$
\begin{gathered}
\left.+k r^{2}-c+5 \sqrt{\Delta}\right) \\
\times\left(2 a\left(\cosh \sqrt{-\frac{\sqrt{\Delta}}{b c}}(x+r y-c t)+1\right)\right)^{-1} .
\end{gathered}
$$

In (18), $\varphi_{1}=\left(1+k r^{2}-c+\sqrt{\Delta}\right) / 2 a$ and $\varphi_{3}=\left(1+k r^{2}-c-\right.$ $2 \sqrt{\Delta}) / 2 a$ if $a<0$ and $b c>0$; then, completing (18) we get the following solution:

$$
\begin{aligned}
& u_{4}(x, y, t) \\
& =\left(\left(1+k r^{2}-c+\sqrt{\Delta}\right)\right.
\end{aligned}
$$

$$
\begin{aligned}
& \left.\times \cosh \sqrt{\frac{\sqrt{\Delta}}{b c}}(x+r y-c t)+1+k r^{2}-c-5 \sqrt{\Delta}\right) \\
& \times\left(2 a\left(\cosh \sqrt{\frac{\sqrt{\Delta}}{b c}}(x+r y-c t)+1\right)\right)^{-1} .
\end{aligned}
$$

Remark 3. If the real number $r$ in (19) and (20) takes value 1 , then solutions (19) and (20) are the same to solutions (1.6) and (1.8) in [6]. Solutions (1.7) and (1.9) in [6] are not real solutions of the KP-BBM equation, and it is easy to verify that they do not satisfy the KP-BBM equation (3). 


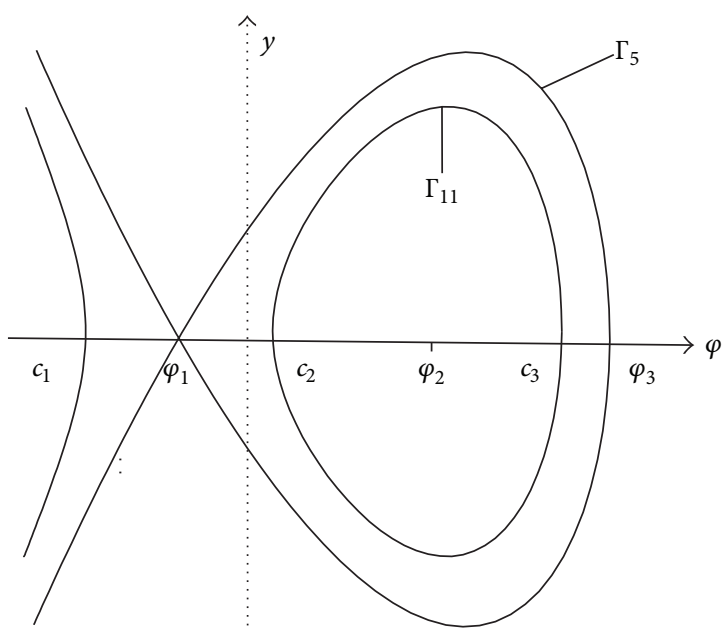

(a) $b c / a<0$

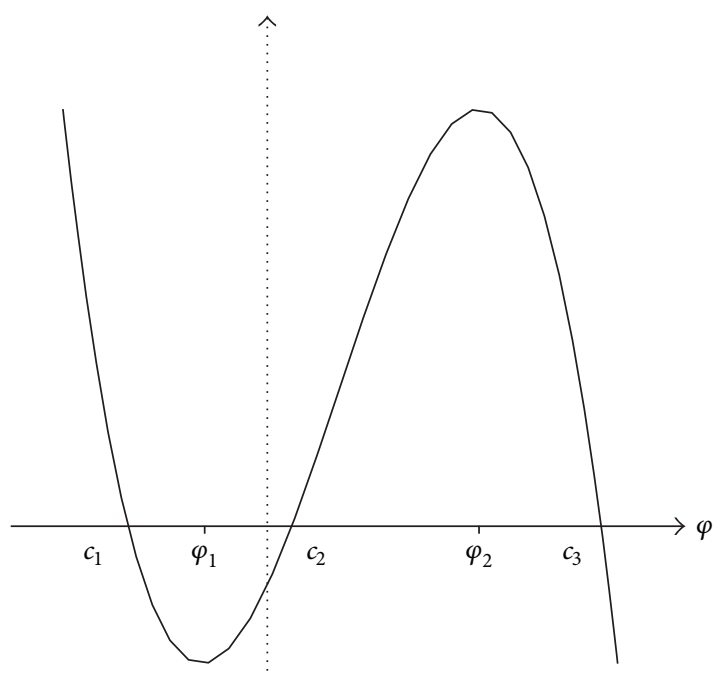

(c)

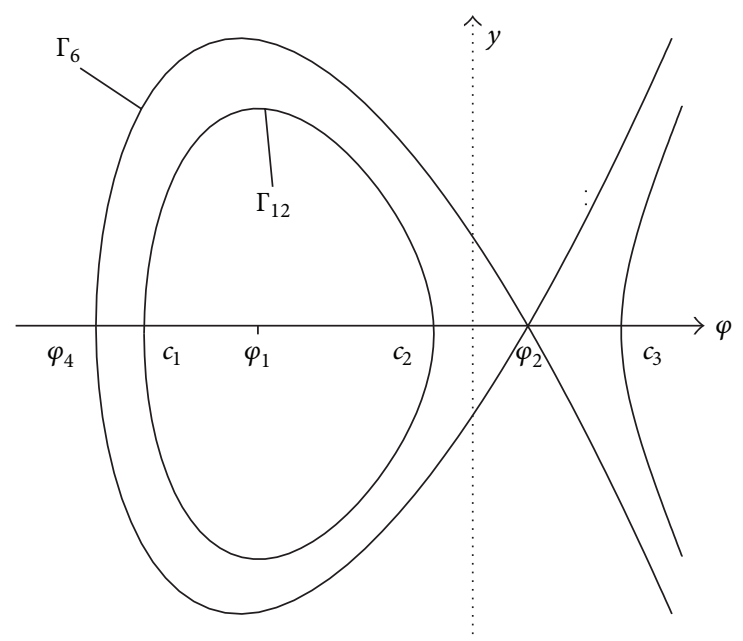

(b) $b c / a>0$

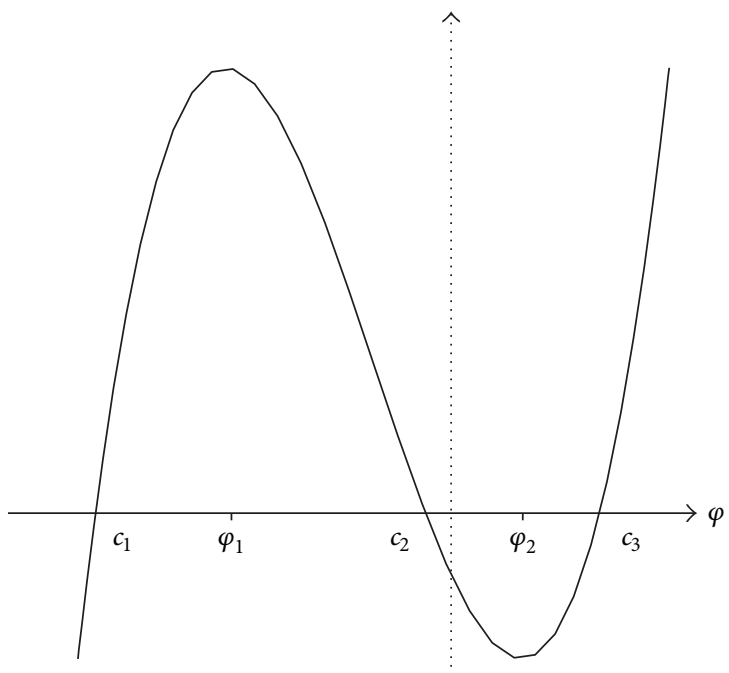

(d)

FIGURE 3: The bifurcation phase portraits of system (6) with $g \neq 0$.

When $b c / a>0$, the corresponding homoclinic orbit $\Gamma_{6}$ has a double zero point $\varphi_{2}$ and a zero point $\varphi_{4}$ on $\varphi$-axis (see Figure 3(b)), so (15) can be rewritten as

$$
y^{2}=\frac{2 a}{3 b c}\left(\varphi-\varphi_{2}\right)^{2}\left(\varphi-\varphi_{4}\right)
$$

that is,

$$
y= \pm \sqrt{\frac{2 a}{3 b c}\left(\varphi-\varphi_{2}\right)^{2}\left(\varphi-\varphi_{4}\right)} .
$$

Substituting (22) into $\mathrm{d} \varphi / \mathrm{d} \xi=y$ and integrating along homoclinic orbits $\Gamma_{6}$, we get

$$
\int_{\varphi_{4}}^{\varphi} \frac{\mathrm{d} s}{\sqrt{(2 a / 3 b c)\left(s-\varphi_{2}\right)^{2}\left(s-\varphi_{4}\right)}}=|\xi|,
$$

where $\varphi_{2}=\left(1+k r^{2}-c-\sqrt{\Delta}\right) / 2 a$ and $\varphi_{4}=\left(1+k r^{2}-c+2 \sqrt{\Delta}\right) / 2 a$ if $a<0$ and $b c<0$; then, completing (23) we get the solution $u_{3}(x, y, t)$. In (23), $\varphi_{2}=\left(1+k r^{2}-c+\sqrt{\Delta}\right) / 2 a$ and $\varphi_{4}=$ $\left(1+k r^{2}-c-2 \sqrt{\Delta}\right) / 2 a$ if $a>0$ and $b c>0$; then, completing (23) we get the solution $u_{4}(x, y, t)$.

\section{Periodic Wave Solutions}

So as to explain our work conveniently, in this section the Jacobian elliptic function $\operatorname{sn}(l, m)$ with modulus $m$ will be expressed by snl. We discuss the periodic wave solutions under conditions $g=0$ and $g \neq 0$, respectively.

4.1. The Case $g=0$. When $\left(1+k r^{2}+c\right) / b c<0$, system (6) has periodic orbits $\Gamma_{7}$ and $\Gamma_{8}$ (see Figures 1(a) and 1(b)). Their 
expressions are (9) on $\varphi$-y plane, where $h_{1}<h<h_{2}$ (or $h_{2}<$ $\left.h<h_{1}\right)$. Let

$$
f_{1}(\varphi)=\frac{2 a}{3 b c} \varphi^{3}-\frac{1+k r^{2}-c}{b c} \varphi^{2}+2 h
$$

then, we have the following results.

Claim 1. In the case of $g=0,\left(1+k r^{2}+c\right) / b c<0$ and $h_{1}<$ $h<h_{2}$ (or $h_{2}<h<h_{1}$ ); then, the function $f_{1}(\varphi)$ must have three different real zero points.

Proof. Since $f_{1}(\varphi)$ is a cubic polynomial about $\varphi$, we can use the Shengjin Theorem [11] to distinguish its solutions. We only discuss the case $b c / a<0$, and the case $b c / a>0$ is the same. Under the above conditions,

$$
\begin{aligned}
& h_{1}=H\left(\varphi_{1}, 0\right)=H(0,0)=0, \\
& h_{2}=H\left(\varphi_{2}, 0\right)=H\left(\frac{1+k r^{2}-c}{a}, 0\right)=\frac{\left(1+k r^{2}-c\right)^{3}}{6 a^{2} b c}<0 .
\end{aligned}
$$

So $\left(1+k r^{2}-c\right) /\left(6 a^{2} b c\right)<h<0$. In $f_{1}(\varphi)$ the coefficients $2 a / 3 b c<0,\left(1+k r^{2}-c\right) / b c<0$, By Shengjing Theorem [11], it follows that the function $f_{1}(\varphi)$ has three different real zero points. Let $A=\left(\left(1+k r^{2}-c\right) / b c\right)^{2}, B=-9(2 a / 3 b c) \cdot 2 h$, and $C=3\left(\left(1+k r^{2}-c\right) / b c\right) \cdot 2 h$; then, $B^{2}-4 A C=144(a / b c)^{2} h[h-$ $\left.\left(\left(1+k r^{2}-c\right)^{3} / 6 a^{2} b c\right)\right]<0$.

Let $r_{1}<r_{2}<r_{3}$ be three different real zero points of $f_{1}(\varphi)$. Then Claim 1 means that $(9)$ has three intersection points $\left(r_{1}, 0\right),\left(r_{2}, 0\right)$, and $\left(r_{3}, 0\right)$ on $\varphi$-axis. Therefore, (9) can be rewritten as

$$
y^{2}=\frac{2 a}{3 b c}\left(\varphi-r_{1}\right)\left(\varphi-r_{2}\right)\left(\varphi-r_{3}\right)
$$

where $r_{1}<0<r_{2}<\varphi<r_{3}$ when $b c / a<0$ and $r_{1}<\varphi<r_{2}<$ $0<r_{3}$ when $b c / a>0$.

When $b c / a<0$, the orbit $\Gamma_{7}$ is according to a periodic solution of (6) and its expression is given by

$$
y= \pm \sqrt{\frac{2 a}{3 b c}\left(\varphi-r_{1}\right)\left(\varphi-r_{2}\right)\left(\varphi-r_{3}\right)}, \quad\left(r_{1}<r_{2} \leq \varphi \leq r_{3}\right) \text {. }
$$

Substituting (27) into $\mathrm{d} \varphi / \mathrm{d} \xi=y$ and integrating along orbit $\Gamma_{7}$, we get

$$
\begin{aligned}
& \int_{\varphi}^{r_{3}} \frac{\mathrm{d} s}{\sqrt{\left(r_{3}-s\right)\left(s-r_{1}\right)\left(s-r_{2}\right)}} \\
& \quad=\sqrt{-\frac{2 a}{3 b c}}|\xi|, \quad\left(r_{1}<r_{2} \leq \varphi<r_{3}\right) .
\end{aligned}
$$

By formula (236) in [12], we have

$$
g_{1} \operatorname{sn}^{-1}\left(\sin \psi_{1}, m_{5}\right)=\sqrt{-\frac{2 a}{3 b c}}|\xi|
$$

where $g_{1}=2 / \sqrt{r_{3}-r_{1}}, \sin \psi_{1}=\sqrt{\left(r_{3}-\varphi\right) /\left(r_{3}-r_{2}\right)}$, and $m_{5}=\sqrt{\left(r_{3}-r_{2}\right) /\left(r_{3}-r_{1}\right)}$. Solving (29), we get

$$
\varphi=r_{3}-\left(r_{3}-r_{2}\right) \operatorname{sn}^{2} \sqrt{-\frac{a\left(r_{3}-r_{1}\right)}{6 b c}} \xi .
$$

That is,

$$
u_{5}(x, y, t)=r_{3}-\left(r_{3}-r_{2}\right) \operatorname{sn}^{2} \sqrt{-\frac{a\left(r_{3}-r_{1}\right)}{6 b c}}(x+r y-c t),
$$

where the modulus of sn is $m_{5}=\sqrt{\left(r_{3}-r_{2}\right) /\left(r_{3}-r_{1}\right)}$.

Similarly, when $b c / a>0$, the expression $\Gamma_{8}$ is

$$
y= \pm \sqrt{\frac{2 a}{3 b c}\left(\varphi-r_{1}\right)\left(\varphi-r_{2}\right)\left(\varphi-r_{3}\right)}, \quad\left(r_{1} \leq \varphi \leq r_{2}<r_{3}\right) \text {. }
$$

Substituting (32) into $\mathrm{d} \varphi / \mathrm{d} \xi=y$ and integrating along orbit $\Gamma_{8}$, we have

$$
\begin{aligned}
& \int_{r_{1}}^{\varphi} \frac{\mathrm{d} s}{\sqrt{\left(r_{3}-s\right)\left(r_{2}-s\right)\left(s-r_{1}\right)}} \\
& \quad=\sqrt{\frac{2 a}{3 b c}}|\xi|, \quad\left(r_{1}<\varphi \leq r_{2}<r_{3}\right) .
\end{aligned}
$$

The according periodic solution of $\Gamma_{8}$ can be obtained as

$$
u_{6}(x, y, t)=r_{1}+\left(r_{2}-r_{1}\right) \operatorname{sn}^{2} \sqrt{\frac{a\left(r_{3}-r_{1}\right)}{6 b c}}(x+r y-c t),
$$

where the modulus of sn is $m_{6}=\sqrt{\left(r_{2}-r_{1}\right) /\left(r_{3}-r_{1}\right)}$.

When $\left(1+k r^{2}+c\right) / b c>0$, system (6) has periodic orbits $\Gamma_{9}$ and $\Gamma_{10}$ (see Figure 2). Their expressions are (9), where $h_{1}<$ $h<h_{2}$ (or $h_{2}<h<h_{1}$ ). Similarly, we can get the according periodic solutions of $\Gamma_{9}$ and $\Gamma_{10}$ as $u_{5}$ and $u_{6}$.

To verify validity of the periodic wave solutions, we take $a=b=k=r=1, c=-1$, and $h=-9 / 4$ to make the conditions in Claim 1 satisfied. By simple calculation, we get that $r_{1}=-1.09808, r_{2}=1.5$, and $r_{3}=4.09808$. The specific periodic wave solution is

$$
u(x, y, t)=4.090808-2.590808 \mathrm{sn}^{2} \sqrt{\frac{5.19616}{6}}(x+y+t),
$$

where the modulus of sn is $\sqrt{2} / 2$.

4.2. The Case $g \neq 0$. System (6) has periodic orbits $\Gamma_{11}$ and $\Gamma_{12}$ (see Figure 3). Their expressions are (7) on the $\varphi$ - $y$ plane, where $h_{1}<h<h_{2}\left(\right.$ or $\left.h_{1}<h<h_{2}\right)$. Let

$$
f_{2}(\varphi)=\frac{2 a}{3 b c} \varphi^{3}-\frac{1+k r^{2}-c}{b c} \varphi^{2}+\frac{2 g}{b c} \varphi+2 h ;
$$

then, we have the following results about $f_{2}(\varphi)$. 
Claim 2. If $g \neq 0$ and $h_{1}<h<h_{2}$ (or $h_{2}<h<h_{1}$ ), then the function $f_{2}(\varphi)$ must have three different real zero points.

Proof. We only prove the case $b c / a<0$, and the case $b c / a>0$ is the same. Under the above conditions,

$$
\begin{aligned}
h_{1} & =H\left(\varphi_{1}, 0\right)=-\frac{a}{3 b c} \varphi_{1}^{3}+\frac{1+k r^{2}-c}{2 b c} \varphi_{1}^{2}-\frac{g}{b c} \varphi_{1} \\
& =-\frac{1}{2} f_{2}\left(\varphi_{1}\right)+h, \\
h_{2} & =H\left(\varphi_{2}, 0\right)=-\frac{a}{3 b c} \varphi_{2}^{3}+\frac{1+k r^{2}-c}{2 b c} \varphi_{2}^{2}-\frac{g}{b c} \varphi_{2} \\
& =-\frac{1}{2} f_{2}\left(\varphi_{2}\right)+h .
\end{aligned}
$$

So $f_{2}\left(\varphi_{1}\right) \cdot f_{2}\left(\varphi_{2}\right)=4\left(h-h_{1}\right)\left(h-h_{2}\right)<0$. For $f_{2}(\varphi)$, we have $f_{2}(-\infty)>0, f_{2}\left(\varphi_{1}\right)<0, f_{2}\left(\varphi_{2}\right)>0$, and $f_{2}(+\infty)<0$. Again, $f_{2}^{\prime}(\varphi)=(2 a / b c)\left(\varphi-\varphi_{1}\right)\left(\varphi-\varphi_{2}\right)$, which is monotonous in the intervals $\left(-\infty, \varphi_{1}\right),\left(\varphi_{1}, \varphi_{2}\right)$, and $\left(\varphi_{2},+\infty\right)$. By zero point theorem of continuous function, there must be one real zero point of $f_{2}(\varphi)$ that lies in each of the three intervals, proving the claim.

Let $c_{1}<c_{2}<c_{3}$ be three different real zero points of $f_{2}(\varphi)$. Then Claim 2 means that (7) has three intersection points on $\varphi$-axis denoted by $\left(c_{1}, 0\right),\left(c_{2}, 0\right)$, and $\left(c_{3}, 0\right)$. Then $(7)$ can be rewritten as

$$
y^{2}=\frac{2 a}{3 b c}\left(\varphi-c_{1}\right)\left(\varphi-c_{2}\right)\left(\varphi-c_{3}\right)
$$

where $c_{1}<\varphi_{1}<c_{2}<\varphi_{2}<c_{3}$.

When $b c / a<0$, the expression of periodic orbit $\Gamma_{11}$ is

$$
y= \pm \sqrt{\frac{2 a}{3 b c}\left(\varphi-c_{1}\right)\left(\varphi-c_{2}\right)\left(\varphi-c_{3}\right)}, \quad\left(c_{1}<c_{2} \leq \varphi \leq c_{3}\right) \text {. }
$$

When $b c / a>0$, the expression of periodic orbit $\Gamma_{12}$ is

$$
y= \pm \sqrt{\frac{2 a}{3 b c}\left(\varphi-c_{1}\right)\left(\varphi-c_{2}\right)\left(\varphi-c_{3}\right)}, \quad\left(c_{1} \leq \varphi \leq c_{2}<c_{3}\right) .
$$

Substituting (39) and (40) into $\mathrm{d} \varphi / \mathrm{d} \xi=y$ and integrating along orbits $\Gamma_{11}$ and $\Gamma_{12}$, respectively, it is the same to the proceeding for solving $u_{5}$ and $u_{6}$ and we can get the according periodic solutions of $\Gamma_{11}$ and $\Gamma_{12}$ as follows:

$$
\begin{aligned}
& u_{7}(x, y, t)=c_{3}-\left(c_{3}-c_{2}\right) \mathrm{sn}^{2} \sqrt{-\frac{a\left(c_{3}-c_{1}\right)}{6 b c}}(x+r y-c t), \\
& u_{8}(x, y, t)=c_{1}+\left(c_{2}-c_{1}\right) s^{2} \sqrt{\frac{a\left(c_{3}-c_{1}\right)}{6 b c}}(x+r y-c t),
\end{aligned}
$$

where the moduli for sn are $m_{7}=\sqrt{\left(c_{3}-c_{2}\right) /\left(c_{3}-c_{1}\right)}$ and $m_{8}=\sqrt{\left(c_{2}-c_{1}\right) /\left(c_{3}-c_{1}\right)}$ in (41).
For example, we take $a=b=k=r=1, c=-1, g=2$, and $h=3 / 2$ such that the conditions in Claim 2 are satisfied. By simple calculation, we get that $c_{1}=0.663975, c_{2}=1.5$, and $c_{3}=2.36603$. The according periodic wave solution is

$$
u(x, y, t)=2.36603-0.86603 \mathrm{sn}^{2} \sqrt{\frac{1.702055}{6}}(x+y+t)
$$

where the modulus of sn is $\sqrt{0.86603 / 1.702055}$.

\section{Relationship between Bell-Shaped Waves and Periodic Waves}

In Sections 3 and 4, the bell-shaped solitary wave and periodic wave solutions are obtained. Via further study, we find that there exists an interesting relationship between these two kinds of solutions; that is, the bell-shaped solutions are limits of the periodic waves in some sense. The results are detailed as follows.

Proposition 4. Let $u_{i}(i=1,2, \ldots, 8)$ be solutions of (3), let $k, r, a, b, c$, and $g$ be parameters in (5), and let $m_{i}(i=$ $5,6,7,8)$ be modulus of the Jacobian elliptic function sn; then, one has the following.

Case 1. When $g=0$ and $\left(1+k r^{2}-c\right) / b c<0$, for modulus $m_{i} \rightarrow 1(i=5,6)$, the periodic waves $u_{5}$ and $u_{6}$ degenerate bell-shaped wave $u_{1}$.

Case 2. When $g=0$ and $\left(1+k r^{2}-c\right) / b c>0$, for modulus $m_{i} \rightarrow 1(i=5,6)$, the periodic waves $u_{5}$ and $u_{6}$ degenerate bell-shaped wave $u_{2}$.

Case 3. When $g \neq 0$ and $b c / a<0$, for modulus $m_{7} \rightarrow 1$, the periodic wave $u_{7}$ degenerates bell-shaped wave $u_{3}$.

Case 4. When $g \neq 0$ and $b c / a>0$, for modulus $m_{8} \rightarrow 1$, the periodic wave $u_{8}$ degenerates bell-shaped wave $u_{4}$.

Here, we only prove Cases 1 and 3 for simplicity. The remaining cases are the same. In the following proofs, we use the property of elliptic function that sn $\rightarrow$ tanh when the modulus $m \rightarrow 1[5,13]$.

Proof of Case 1. When $m_{5}=\sqrt{\left(r_{3}-r_{2}\right) /\left(r_{3}-r_{1}\right)} \rightarrow 1$, it means $r_{1}=r_{2}$ and $s n=$ tanh; then, we calculate

$$
r_{1}=r_{2}=0, \quad r_{3}=\frac{3\left(1+k r^{2}-c\right)}{2 a} .
$$

Substituting $r_{i}(i=1,2,3)$ into $u_{5}$ admits to $u_{1}$ as follows:

$$
\begin{aligned}
& u_{5}(x, y, t) \\
& =r_{3}-\left(r_{3}-r_{2}\right) \operatorname{sn}^{2} \sqrt{-\frac{a\left(r_{3}-r_{1}\right)}{6 b c}(x+r y-c t)} \\
& =\frac{3\left(1+k r^{2}-c\right)}{2 a}-\frac{3\left(1+k r^{2}-c\right)}{2 a}
\end{aligned}
$$




$$
\begin{aligned}
& \times \tanh ^{2} \sqrt{-\frac{\left(1+k r^{2}-c\right)}{4 b c}}(x+r y-c t) \\
& =\frac{3\left(1+k r^{2}-c\right)}{2 a} \\
& \times\left[1-\tanh ^{2} \sqrt{-\frac{\left(1+k r^{2}-c\right)}{4 b c}}(x+r y-c t)\right] \\
& =\frac{3\left(1+k r^{2}-c\right)}{2 a} \\
& \times \frac{1}{\cosh ^{2} \sqrt{-\left(\left(1+k r^{2}-c\right) / 4 b c\right)}(x+r y-c t)} \\
& =\frac{3\left(1+k r^{2}-c\right)}{2 a} \\
& \times \frac{1}{(1 / 2)\left[\cosh \sqrt{-\left(\left(1+k r^{2}-c\right) / b c\right)}(x+r y-c t)+1\right]} \\
& =\frac{3\left(1+k r^{2}-c\right)}{a\left[1+\cosh \sqrt{-\left(\left(1+k r^{2}-c\right) / b c\right)}(x+r y-c t)\right]} \\
& =u_{1}(x, y, t) \text {. }
\end{aligned}
$$

When $m_{6}=\sqrt{\left(r_{2}-r_{1}\right) /\left(r_{3}-r_{1}\right)} \rightarrow 1$, it means $r_{2}=r_{3}$; then, we calculate $r_{2}=r_{3}=0$ and $r_{1}=3\left(1+k r^{2}-c\right) / 2 a$, and substituting $r_{i}(i=1,2,3)$ into $u_{6}$ we get $u_{6}=u_{1}$.

Proof of Case 3. When $m_{7}=\sqrt{\left(c_{3}-c_{2}\right) /\left(c_{3}-c_{1}\right)} \rightarrow 1$, it means $c_{1}=c_{2}$ and $\mathrm{sn}=\tanh$; then, we calculate $c_{1}=c_{2}=$ $\left(1+k r^{2}-c-\sqrt{\Delta}\right) / 2 a$ and $c_{3}=\left(1+k r^{2}-c+2 \sqrt{\Delta}\right) / 2 a$, and substituting $c_{i}(i=1,2,3)$ into $u_{7}$ admits to $u_{3}$ as follows:

$$
\begin{aligned}
u_{7}(x, y, t) & c_{3}-\left(c_{3}-c_{2}\right) \operatorname{sn}^{2} \sqrt{-\frac{a\left(c_{3}-c_{1}\right)}{6 b c}}(x+r y-c t) \\
= & \frac{1+k r^{2}-c+2 \sqrt{\Delta}}{2 a}-\frac{3 \sqrt{\Delta}}{2 a} \tanh ^{2} \sqrt{\frac{-\sqrt{\Delta}}{4 b c}}(x+r y-c t) \\
= & \left(1+k r^{2}-c-\sqrt{\Delta}\right. \\
& \left.+3 \sqrt{\Delta}\left(1-\tanh ^{2} \sqrt{\frac{-\sqrt{\Delta}}{4 b c}}(x+r y-c t)\right)\right) \\
& \times(2 a)^{-1}
\end{aligned}
$$

$$
\begin{aligned}
= & \frac{1+k r^{2}-c-\sqrt{\Delta}}{2 a} \\
& +\frac{3 \sqrt{\Delta}}{a[1+\cosh \sqrt{-\sqrt{\Delta} / b c}(x+r y-c t)]} \\
= & \left(1+k r^{2}-c-\sqrt{\Delta}\right) \\
& \times \cosh \sqrt{-\frac{\sqrt{\Delta}}{b c}(x+r y-c t)+1} \\
& \left.+k r^{2}-c+5 \sqrt{\Delta}\right) \\
& \times\left(2 a\left[\cosh \sqrt{-\frac{\sqrt{\Delta}}{b c}}(x+r y-c t)+1\right]\right)^{-1} \\
= & u_{3}(x, y, t) .
\end{aligned}
$$

The results provide a manner that we can get bell-shaped waves from periodic waves for some nonlinear development equations.

\section{Conflict of Interests}

The author declares that there is no conflict of interests regarding the publication of this paper.

\section{Acknowledgments}

This work was supported by the National Natural Science Foundation of China (no. 11201070) and Guangdong Province (no. 2013KJCX0189 and no. Yq2013161). The author would like to thank the editors for their hard working and the anonymous reviewers for helpful comments and suggestions.

\section{References}

[1] T. B. Benjamin, J. L. Bona, and J. J. Mahony, "Model equations for long waves in nonlinear dispersive systems," Philosophical Transactions of the Royal Society of London A. Mathematical and Physical Sciences, vol. 272, no. 1220, pp. 47-78, 1972.

[2] B. B. Kadomtsev and V. I. Petviashvili, "On the stability of solitary waves in weakly dispersive media," Soviet PhysicsDoklady, vol. 15, pp. 539-541, 1970.

[3] A.-M. Wazwaz, "Exact solutions of compact and noncompact structures for the KP-BBM equation," Applied Mathematics and Computation, vol. 169, no. 1, pp. 700-712, 2005.

[4] A.-M. Wazwaz, "The extended tanh method for new compact and noncompact solutions for the KP-BBM and the ZK-BBM equations," Chaos, Solitons and Fractals, vol. 38, no. 5, pp. 15051516, 2008. 
[5] M. A. Abdou, "Exact periodic wave solutions to some nonlinear evolution equations," International Journal of Nonlinear Science, vol. 6, no. 2, pp. 145-153, 2008.

[6] M. Song, C. X. Yang, and B. G. Zhang, "Exact solitary wave solutions of the Kadomtsov-Petviashvili-Benjamin-Bona-Mahony equation," Applied Mathematics and Computation, vol. 217, no. 4, pp. 1334-1339, 2010.

[7] S.-N. Chow and J. K. Hale, Method of Bifurcation Theory, Springer, Berlin, Germany, 1982.

[8] J. B. Li and Z. R. Liu, "Smooth and non-smooth traveling waves in a nonlinearly dispersive equation," Applied Mathematical Modelling, vol. 25, no. 1, pp. 41-56, 2000.

[9] J. B. Li and Z. R. Liu, "Traveling wave solutions for a class of nonlinear dispersive equations," Chinese Annals of Mathematics B, vol. 23, no. 3, pp. 397-418, 2002.

[10] Z. R. Liu and Z. Y. Ouyang, "A note on solitary waves for modified forms of Camassa-Holm and Degasperis-Procesi equations," Physics Letters A, vol. 366, no. 4-5, pp. 377-381, 2007.

[11] F. Shengjin, "A new extracting formula and a new distinguishing means on the one variable cubic equation," Natural Science Journal of Hainan Teacheres College, vol. 2, pp. 91-98, 1989.

[12] P. F. Byrd and M. D. Friedman, Handbook of Elliptic Integrals for Engineers and Scientists, Springer, Berlin, Germany, 1971.

[13] S. Yu and L. Tian, "Nonsymmetrical kink solution of generalized KdV equation with variable coefficients," International Journal of Nonlinear Science, vol. 5, no. 1, pp. 71-78, 2008. 


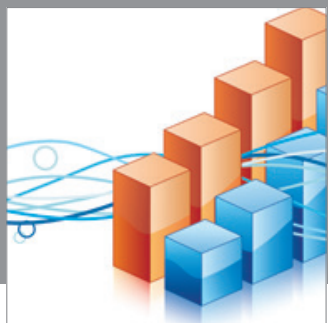

Advances in

Operations Research

mansans

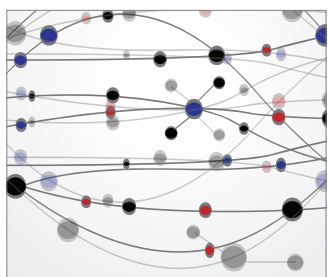

The Scientific World Journal
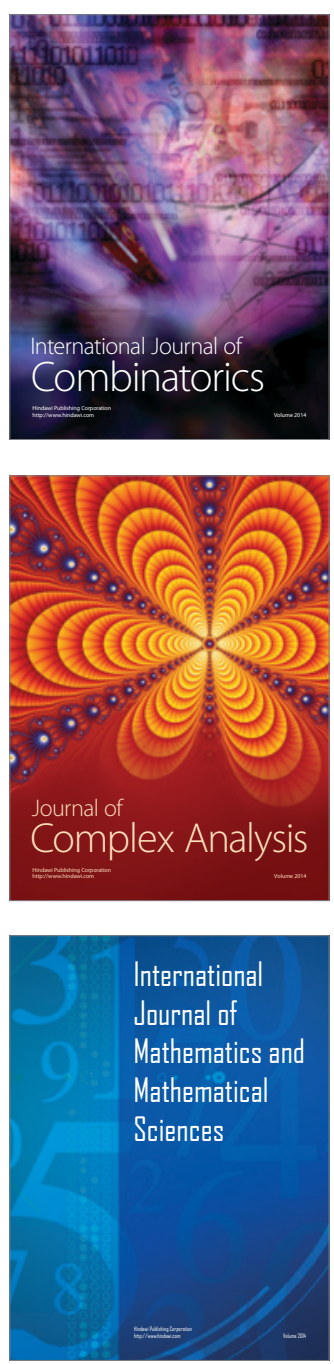
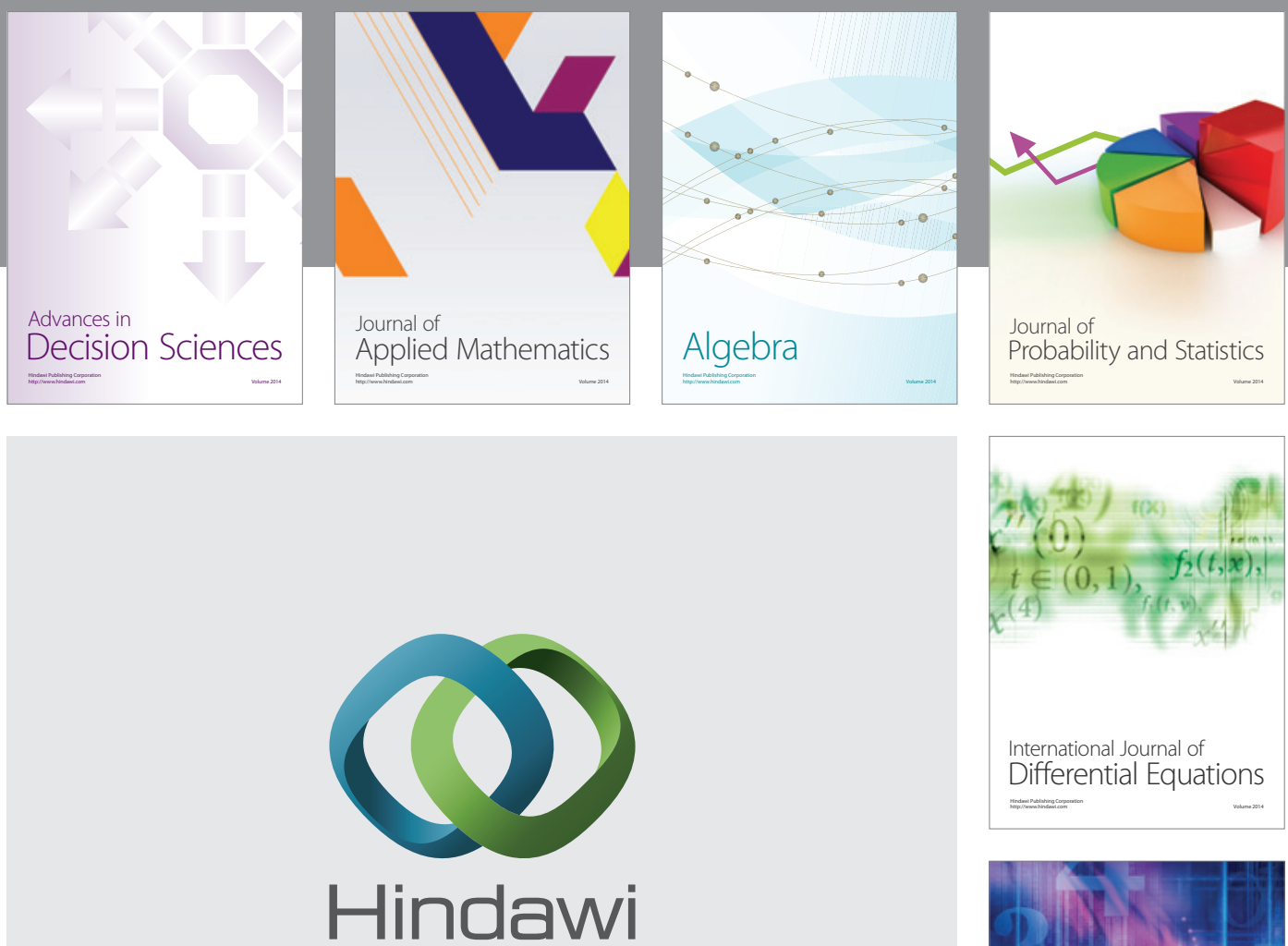

Submit your manuscripts at http://www.hindawi.com
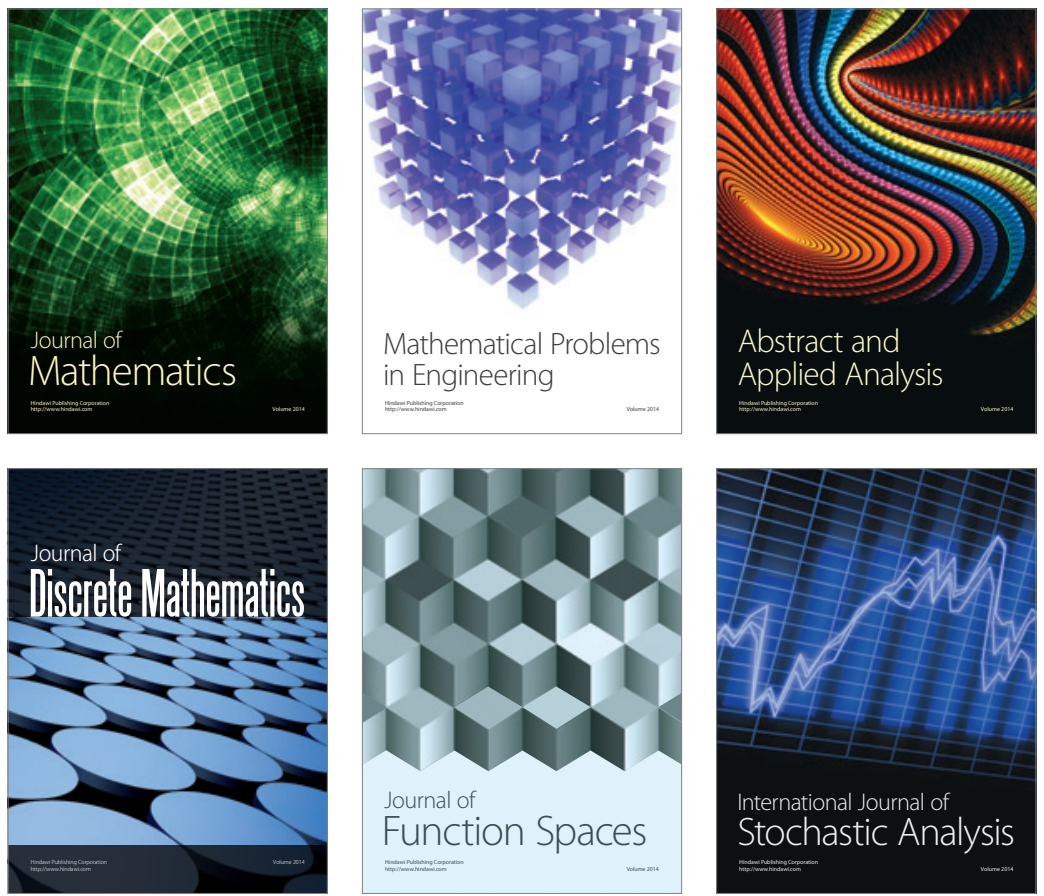

Journal of

Function Spaces

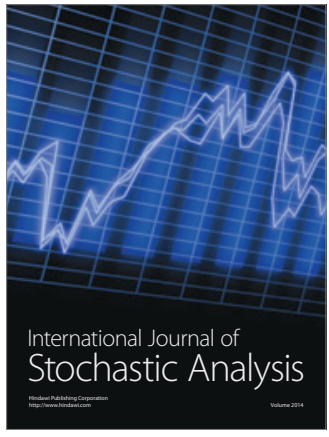

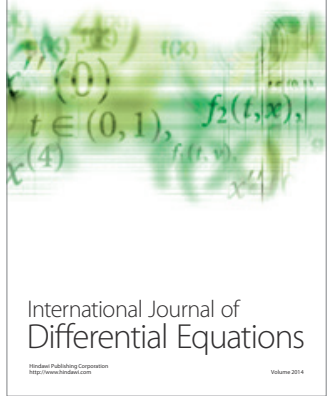
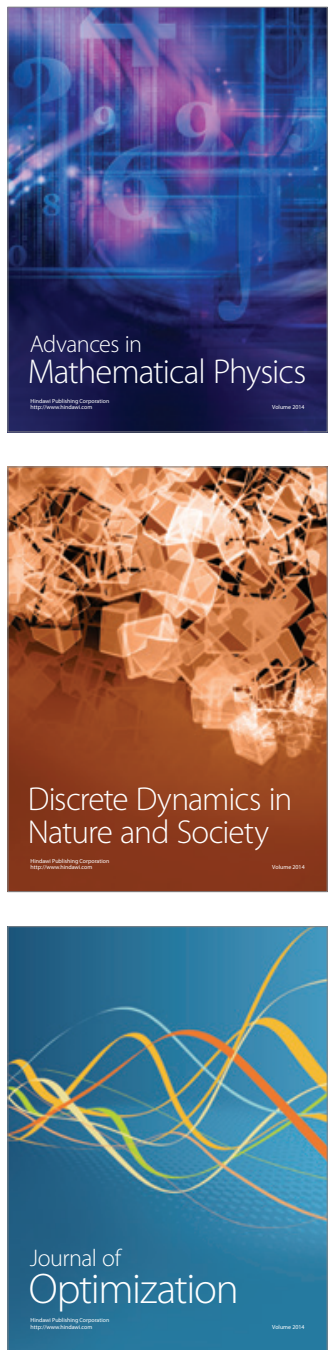